\title{
A commentary on a case of new $P C D H 12$ gene variants presented as dyskinetic cerebral palsy with epilepsy
}

\author{
Eraldo Fonseca dos Santos-Junior ${ }^{1}$ - João Ricardo Mendes de Oliveira ${ }^{2}$
}

Received: 30 April 2018 / Revised: 4 May 2018 / Accepted: 16 May 2018 / Published online: 14 June 2018

(c) The Author(s) under exclusive licence to The Japan Society of Human Genetics 2018

In the last 2 years, literature data have shown that variations in protocadherin 12 ( $\mathrm{PCDH12}$ ) gene are related to neurological outcomes. Located at chromosome 5q31, this gene encodes the PCDH12 protein, wich is part of the cadherin superfamily and it is a calcium-dependent cell-adhesion protein. The role of PCDH12 is not yet fully understood in the brain, but evidence shows the importance of nonclustered protocadherins for the normal development of the central nervous system [1].

In a recent publication in this journal, Suzuki-Muromoto et al. reported a case of a Japanese patient with dyskinetic cerebral palsy, intellectual disability and epilepsy, born to nonconsanguineous parents, with two heterozygous variations in the $P C D H 12$ gene, a different pattern from the other cases already described [2]. The first work that showed a relation of a variation in the $P C D H 12$ gene with neurological outcomes was published by Aran et al., where Palestinian patients from consanguineous families, developed pre- and postnatal damages, with a pseudo-TORCH pattern, from a single homozygous nonsense variation [3]. The homozygous variation described, occurred in the cytoplasmic domain of the PCDH12 protein, while the double heterozygous variation occurred in the extracellular domain (Fig. 1).

Knockout mice for the $P C D H 12$ gene are alive and fertile, but, present alterations in the placenta in relation to their morphogenesis, vascularization and transcriptional levels of 2289 genes, contributing to a reduced embryo size and abnormal lens morphology [4]. Different from that reported in humans, in mice, only the homozygous variation is able to develop phenotypes harmful to the animal, heterozygous variation did not cause any harmful consequences.

João Ricardo Mendes de Oliveira

joao.ricardo@ufpe.br

1 Keizo Asami Laboratory - Federal University of Pernambuco, Recife-PE, Brazil

2 Federal University of Pernambuco, Recife-PE, Brazil
In humans, the condition of two heterozygous variations did not result in intrauterine growth retardation, microcephaly and visual impairment, clinical findings of patients with homozygous variation. Other neurological findings are shared among patients with variations in the PCDH12 gene, regardless of the mutation pattern, such as cerebral calcification and epilepsy. Spot calcifications in perithalamic regions are found in some patients already reported, but other areas, particularly in each patient described, also had a spot calcification, such as subcortical regions and posterior limb of internal capsule [5]. Figure 1 also shows heterozygous variants, with no clear causative role, found in some elder subjects with primary brain calcification, presenting with late and variable manifestations such as dementia and gait impairment.

The brain has a very low expression of PCDH12 [6], therefore, the amount of negative effects that happens when this gene is mutated calls attention. Although there are few cases reporting variations of this gene, what is perceived is that the mutation pattern seems to influence the expression of certain phenotypes and consequent severity of the patient. As already discussed here and as can be seen in table 1 of the article by Suzuki-Muromoto et al. (2018), in general, homozygous variation seems to be more damaging to the patient, especially in relation to the development and onset of seizures.

The evaluation of transcript expression of $\mathrm{PCDH} 12$ would bring more data to increase discussion and knowledge of the impact of two heterozygous variants in the described patient. The patient is the fourth child of healthy parents, and for a better description of the finding, a family segregation analysis would be an important contribution to the study of the variations of this gene. Beyond the mutation pattern, environmental factors should also be considered for future analysis of the differences between the phenotypes developed by the patients.

In conclusion, the identification of a new mutation pattern of the $P C D H 12$ gene, brings to light new clinical findings that may help better understanding the role of this gene in the central nervous system. His role is still intriguing, mainly because it is related to distinct brain areas and the development of diverse clinical findings. The use of animal models presents 


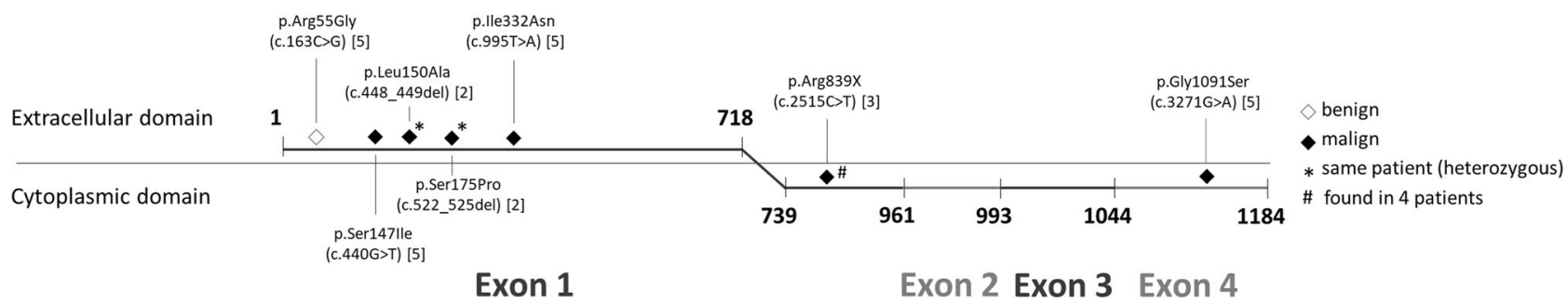

Fig. 1 Distribution of variations along the $\mathrm{PCDH} 12$ gene. The numbers in brackets identify the reference number

a good opportunity for the understanding of this gene, models that already exist and that need to be more explored from the neurological point of view, pre- and postnatal.

\section{Compliance with ethical standards}

Conflict of interest The authors declare that they have no conflict of interest.

\section{References}

1. Kim SY, Yasuda S, Tanaka H, Yamagata K, Kim H. Non-clustered protocadherin. Cell Adhes Migr. 2011;5:97-105.
2. Suzuki-Muromoto S, Wakusawa K, Miyabayashi T, Sato R, Okubo Y, Endo W, et al. A case of new PCDH12 gene variants presented as dyskinetic cerebral palsy with epilepsy. J Hum Genet. 2018;63:749-53.

3. Aran A, Rosenfeld N, Jaron R, Renbaum P, Zuckerman S, Fridman H, et al. Loss of function of PCDH12 underlies recessive microcephaly mimicking intrauterine infection. Neurology. 2016;86:2016-24.

4. Rampon C, Bouillot S, Climescu-Haulica A, Prandini M-H, Cand F, Vandenbrouck Y, et al. Protocadherin 12 deficiency alters morphogenesis and transcriptional profile of the placenta. Physiol Genom. 2008;34:193-204.

5. Nicolas G, Sanchez-Contreras M, Ramos EM, Lemos RR, Ferreira $\mathrm{J}$, Moura D, et al. Brain calcifications and $\mathrm{PCDH} 12$ variants. Neurol Genet. 2017;3:e166.

6. Philibert C, Bouillot S, Huber P, Faury G. Protocadherin-12 deficiency leads to modifications in the structure and function of arteries in mice. Pathol Biol. 2012;60:34-40. 